\title{
Analysis of Energy Detection of Unknown Signals under Beckmann Fading Channels
}

\author{
Juan P. Peña-Martin, \\ Dept. Electronic Technology \\ University of Málaga \\ 29071 Málaga, Spain \\ Email: jppena@uma.es
}

\author{
Juan M. Romero-Jerez \\ Dept. Electronic Technology \\ University of Málaga \\ 29071 Málaga, Spain \\ Email: romero@dte.uma.es
}

\author{
F. Javier Lopez-Martinez \\ Dept. Communication Engineering \\ University of Málaga \\ 29071 Málaga, Spain \\ Email: fjlopezm@ic.uma.es
}

\begin{abstract}
The Beckmann fading is a general multipath fading model which includes Rice, Hoyt and Rayleigh fading as particular cases. However, the generality of the Beckmann fading also implies a significant increased mathematical complexity. Thus, relatively few analytical results have been reported for this otherwise useful fading model. The performance of energy detection for multi-branch receivers operating under Beckmann fading is here studied, and the inherent analytical complexity is here circumvented by the derivation of a closed-form expression for the generalized moment generating function (MGF) of the received signal-to-noise ratio (SNR), which is a new and useful result, as it is key for evaluating the receiver operating characteristics. The impact of fading severity on the probability of missed detection is shown to be less critical as the SNR decreases. Monte Carlo simulations have been carried out in order to validate the obtained theoretical expressions.
\end{abstract}

\section{INTRODUCTION}

The need of a more efficient use of radio spectrum has boosted the research on cognitive radio systems in the last decade [1]. In such scenarios, the search for unused channels on which opportunistically transmit without causing or experiencing interference is of paramount relevance for a proper system operation [2]. Under these conditions, the reliable detection of user activity or inactivity in a certain time or frequency resource becomes critical.

The problem of detecting a signal of unknown form in the presence of noise is a classical problem in communication theory, ever since the detection of unknown but deterministic signals using an energy-measuring device was studied by Urkowitz [3]. However, the effect of multipath fading clearly has an impact on the detection performance as it introduces a random fluctuation on the signal of interest. This was addressed in [4], on which the performance of energy detection techniques in Rayleigh and Nakagami- $m$ fading channels was evaluated. In this milestone work, several diversity combining strategies were proposed, which showed a significant performance improvement over no-diversity schemes even in the presence of branch correlation.

In the last years, the performance evaluation of energy detection schemes operating in more sophisticated fading scenarios has been a hot research topic. Some examples include Rice, Hoyt [5], $\kappa-\mu$ [6], $\eta-\mu$ [7], hyper-Rayleigh [8] and cascaded fading channels $[9,10]$ just to name a few. While these works illustrate the interest on knowing the effect of different fading conditions on the performance of energy detectors, the consideration of more general fading models usually comes at the price of increased mathematical complexity.

A very general fading distribution was introduced by Beckmann to characterize scattering from rough surfaces [11] in the form of a four-parameter distribution modeling the envelope of a complex Gaussian random variable (RV) with arbitrary mean and variance for both the in-phase and quadrature components. This distribution includes Rice, Hoyt and Rayleigh fading models as particular cases. Besides, it allows for modeling line-of-sight (LOS) propagation conditions with a Hoyt-distributed diffuse component, which accurately fit field measurements in different scenarios [12-14]. Despite being very general, the fact that its probability density function (PDF) and cumulative probability function (CDF) have integral form clearly hindered the performance evaluation of wireless communication systems operating this otherwise intuitive and physically-justified fading model. Remarkable efforts have been made in order to analyze different performance metrics under Beckmann fading [15-17], but the analysis of energy detection in this scenario is still largely unknown.

In this work, we aim to fill this gap by analyzing the performance of multi-branch energy detection receivers in the presence of Beckmann fading. In the course of our derivations, we obtain an exact and closed-form expression for the generalized MGF of the received signal-to-noise ratio (SNR) under Beckmann fading in terms of a finite number of elementary functions, which is also a new and useful result. Interestingly, the expressions derived herein have similar complexity to those obtained for the Rician or Hoyt cases.

The remainder of this paper is organized as follows. In Section II we describe the channel model and introduce some definitions, whereas the main mathematical contributions are included in Section III. Section IV uses these results to calculate the average detection probability under squarelaw combining (SLC) and maximal ratio combining (MRC) strategies. Numerical results are given in Section V, whereas concluding remarks are presented in Section VI. 


\section{Preliminary Definition and Channel Model}

We present some definitions which will be of later use in the analysis.

Definition 1 (Generalized MGF): Let $\xi$ be a continuous random variable with $\mathrm{PDF} f_{\xi}(\cdot)$. The generalized MGF of $\xi$ is defined as

$$
\phi_{\xi}^{(n)}(s) \triangleq E\left\{\xi^{n} e^{\xi s}\right\}=\int_{0}^{\infty} x^{n} e^{x s} f_{\xi}(x) d x,
$$

where $E\{\cdot\}$ denotes the expectation operator.

In the sequel, we will assume $n \in \mathbb{N}$. Note that in this case the generalized MGF coincides with the $n^{\text {th }}$ order derivative of the $\operatorname{MGF} \phi_{\xi}(s) \triangleq E\left\{e^{\xi s}\right\}=\phi_{\xi}^{(0)}(s)$.

Definition 2 (Beckmann envelope distribution): Let $v=X+j Y$ be a complex Gaussian RV such as $X \sim \mathcal{N}\left(\mu_{x}, \sigma_{x}^{2}\right)$ and $Y \sim \mathcal{N}\left(\mu_{y}, \sigma_{y}^{2}\right)$, being $X$ and $Y$ independent. Then, the RV $R=|v|$ representing the signal envelope, is said to be Beckmann distributed and its PDF is given by [18, eq. (31)]

$$
f_{V}(v)=\frac{v}{2 \pi \sigma_{x} \sigma_{y}} \int_{0}^{2 \pi} e^{-\frac{\left(v \cos (\theta)-\mu_{x}\right)^{2}}{2 \sigma_{x}^{2}}-\frac{\left(v \sin (\theta)-\mu_{y}\right)^{2}}{2 \sigma_{y}^{2}}} d \theta
$$

The Beckmann distribution includes the most popular fading distributions such as Rayleigh, Hoyt and Rician as particular cases, by simply specializing the four parameters $\mu_{x}, \mu_{y}, \sigma_{x}$ and $\sigma_{y}$. For convenience of discussion, an alternative definition for the parameters of the Beckmann distribution is usually preferred [19, eq. (2.39)].

Definition 3 (Beckmann distribution parameters): Let $R$ be a Beckmann distributed RV with parameters $\mu_{x}, \mu_{y}, \sigma_{x}^{2}$ and $\sigma_{y}^{2}$. Then, the following parameters are defined in order to univoquely define the Beckmann distribution:

$$
\begin{aligned}
& q^{2} \triangleq \frac{\sigma_{x}^{2}}{\sigma_{y}^{2}}, \quad r^{2} \triangleq \frac{\mu_{x}^{2}}{\mu_{y}^{2}}, \quad K \triangleq \frac{\mu_{x}^{2}+\mu_{y}^{2}}{\sigma_{x}^{2}+\sigma_{y}^{2}}, \\
& \Omega \triangleq \mu_{x}^{2}+\mu_{y}^{2}+\sigma_{x}^{2}+\sigma_{y}^{2} .
\end{aligned}
$$

The parameter $K$ accounts for the ratio between the LOS and non-LOS (NLOS) power, similarly to the definition of the Rician $K$ parameter. In the same way, the parameter $q$ measures the power imbalance between the in-phase (I) and quadrature $(\mathrm{Q})$ NLOS components as in the Hoyt (Nakagami$q$ ) fading model. The parameter $r$ also indicates a power imbalance between the I and Q components, but now for the LOS component ${ }^{1}$. Finally the parameter $\Omega$ can be regarded as the average received power $\Omega=E\left\{|v|^{2}\right\}=E\left\{R^{2}\right\}$. The connection between the Beckmann distribution and the special cases included therein can easily be set by using the previous definitions for $q, r$ and $K$, and is formally stated in Table I.

\footnotetext{
${ }^{1}$ Note that the parameters $q$ and $r$ can take values within the whole range $[0, \infty)$, showing a symmetric behavior in the intervals $[0,1]$ and $[1, \infty)$.
}

Table I

\begin{tabular}{|c|c|}
\hline Channels & Beckmann Fading Parameters \\
\hline One-sided Gaussian & $\underline{r}=1, \quad \underline{q}=0, \quad \underline{K}=0$ \\
\hline Rayleigh & $\underline{r}=1, \quad \underline{q}=1, \quad \underline{K}=0$ \\
\hline Hoyt (Nakagami- $q$ ) & $\underline{r}=1, \quad \underline{q}=q, \quad \underline{K}=0$ \\
\hline Rician with parameter $K$ & $\underline{r}=1, \quad \underline{q}=1, \quad \underline{K}=K$ \\
\hline LOS with diffuse Hoyt & $\underline{r}=1, \quad \underline{q}=q, \quad \underline{K}=K$ \\
\hline Symmetrical $\eta-\kappa[20]$ & $\underline{r}=\eta, \quad \underline{q}=\eta, \quad \underline{K}=\kappa$ \\
\hline Asymmetrical $\eta-\kappa[21]$ & $\underline{r}=0, \quad q=\eta, \quad \underline{K}=\kappa$ \\
\hline
\end{tabular}

CONNECTIONS BETWEEN BECKMANN FADING AND OTHER FADING MODELS IN THE LITERATURE. THE BECKMANN FADING PARAMETERS ARE UNDERLINED TO AVOID CONFUSION WITH THE SPECIAL CASES.

\section{Mathematical Results}

We now present the main mathematical contributions of this work, which will be of use in the forthcoming derivations.

Lemma 1: Let $R$ be a Beckmann distributed RV with $E\left\{R^{2}\right\}=\Omega$. Let $\gamma \triangleq R^{2} E_{s} / N_{0}$ be the received SNR under Beckmann fading, where $E_{s}$ is the symbol energy and $N_{0}$ is the one-sided AWGN power spectral density, and let $\bar{\gamma}=\Omega E_{s} / N_{0}$ denote its average. Then, the generalized MGF of $\gamma, \phi_{\gamma}^{(n)}(s)$, is given by (5).

Proof: Let $R^{2}$ denote the power envelope of the Beckmann distribution, i.e., $R^{2} \triangleq X^{2}+Y^{2}$ such that $X \sim$ $\mathcal{N}\left(\mu_{x}, \sigma_{x}^{2}\right)$ and $Y \sim \mathcal{N}\left(\mu_{y}, \sigma_{y}^{2}\right)$, being $X$ and $Y$ independent. Both $X^{2}$ and $Y^{2}$ follow a non-central chi-squared distribution, which PDF is given by [22, eq. (2.1-115)]

$$
f(z)=\frac{1}{\sqrt{2 \pi z} \sigma_{i}} \exp \left[\frac{-\left(z+\mu_{i}^{2}\right)}{2 \sigma_{i}^{2}}\right] \cosh \left(\frac{\mu_{i} \sqrt{z}}{\sigma_{i}^{2}}\right), z \geq 0,
$$

where the subindex $i$ denotes either $x$ or $y$.

Given that $R^{2 n}=\sum_{k=0}^{n}\left(\begin{array}{l}n \\ k\end{array}\right) X^{2 k} Y^{2(n-k)}$ and assuming the independence of $X$ and $Y$, the desired expected value is obtained by averaging over both distributions, considering that each one of them follows (6), yielding,

$$
\begin{aligned}
& E_{R^{2}}\left\{R^{2 n} e^{s R^{2}}\right\}=\sum_{k=0}^{n}\left(\begin{array}{l}
n \\
k
\end{array}\right) E\left\{X^{2 k} e^{s X^{2}}\right\} E\left\{Y^{2(n-k)} e^{s Y^{2}}\right\} \\
& =\frac{1}{2 \pi \sigma_{x} \sigma_{y}} \sum_{k=0}^{n}\left(\begin{array}{l}
n \\
k
\end{array}\right) \\
& \times \int_{0}^{\infty} y_{1}^{k-1 / 2} \exp \left[s y_{1}-\frac{y_{1}+\mu_{x}^{2}}{2 \sigma_{x}^{2}}\right] \cosh \left(\frac{\mu_{x} \sqrt{y_{1}}}{\sigma_{x}^{2}}\right) d y_{1} \\
& \times \int_{0}^{\infty} y_{2}^{n-k-1 / 2} \exp \left[s y_{2}-\frac{y_{2}+\mu_{y}^{2}}{2 \sigma_{y}^{2}}\right] \cosh \left(\frac{\mu_{y} \sqrt{y_{2}}}{\sigma_{y}^{2}}\right) d y_{2} .
\end{aligned}
$$




$$
\begin{aligned}
& \phi_{\gamma}^{(n)}(s)=\frac{\left(1+q^{2}\right)(1+K)}{\sqrt{\left[\left(1+q^{2}\right)(1+K)-2 q^{2} \bar{\gamma} s\right]\left[\left(1+q^{2}\right)(1+K)-2 \bar{\gamma} s\right]}} \\
& \times \exp \left[\frac{K \frac{1}{1+r^{2}}\left(1+q^{2}\right) \bar{\gamma} s}{\left(1+q^{2}\right)(1+K)-2 \bar{\gamma} s}+\frac{K \frac{r^{2}}{1+r^{2}}\left(1+q^{2}\right) \bar{\gamma} s}{\left(1+q^{2}\right)(1+K)-2 q^{2} \bar{\gamma} s}\right] \\
& \times \bar{\gamma}^{n} \frac{n !}{2^{n}} \sum_{k=0}^{n} \frac{(2 k) !(2(n-k)) !}{k !(n-k) !}\left(\frac{q^{2}}{\left(1+q^{2}\right)(1+K)-2 q^{2} \bar{\gamma} s}\right)^{k}\left(\frac{1}{\left(1+q^{2}\right)(1+K)-2 \bar{\gamma} s}\right)^{n-k} \\
& \times\left[\sum_{m=0}^{k} \frac{1}{(k-m) !(2 m) !}\left(\frac{\frac{2 r^{2}}{1+r^{2}} \frac{\left(1+q^{2}\right)^{2}}{q^{2}} K(1+K)}{\left(1+q^{2}\right)(1+K)-2 q^{2} \bar{\gamma} s}\right)^{m}\right]\left[\sum_{m=0}^{n-k} \frac{1}{(n-k-m) !(2 m) !}\left(\frac{\frac{2}{\left(1+r^{2}\right)}\left(1+q^{2}\right)^{2} K(1+K)}{\left(1+q^{2}\right)(1+K)-2 \bar{\gamma} s}\right)^{m}\right]
\end{aligned}
$$

In order to solve these integrals it is convenient to calculate the Laplace transform

$$
\begin{aligned}
F(z) & =\mathfrak{L}\left\{x^{m-1 / 2} \cosh (a \sqrt{x}) ; z\right\} \\
& =\frac{1}{2} \int_{0}^{\infty} x^{m-1 / 2} e^{-z x}\left[e^{a \sqrt{x}}+e^{-a \sqrt{x}}\right] d x \\
& =\int_{0}^{\infty} y^{2 m} \exp \left[-z y^{2}+a y\right] d y \\
& +\int_{0}^{\infty} y^{2 m} \exp \left[-z y^{2}-a y\right] d y,
\end{aligned}
$$

where we have made the change of variables $y=\sqrt{x}$. Now, let us introduce [23, eq. (3.462.1)]

$$
\begin{aligned}
& \int_{0}^{\infty} y^{\nu-1} \exp \left[-\beta y^{2}-\alpha y\right] d y \\
& \quad=(2 \beta)^{-\nu / 2} \Gamma(\nu) \exp \left[\frac{\alpha^{2}}{8 \beta}\right] D_{-\nu}\left(\frac{\alpha}{\sqrt{2 \beta}}\right) ; \\
& \Re(\nu)>0 \wedge \Re(\beta)>0,
\end{aligned}
$$

where $\Re(z)$ denotes the real part of complex number $z$, and $D_{\nu}(z)$ is the parabolic-cylinder function defined in [23, eq. (9.240)] as

$$
\begin{aligned}
D_{\nu}(z) & =2^{\nu / 2} e^{-z^{2} / 4} \sqrt{\pi}\left[\frac{1}{\Gamma\left(\frac{1-\nu}{2}\right)}{ }_{1} F_{1}\left(\frac{-\nu}{2} ; \frac{1}{2} ; \frac{z^{2}}{2}\right)\right. \\
& \left.-\frac{a \sqrt{2}}{\Gamma\left(\frac{-\nu}{2}\right)}{ }_{1} F_{1}\left(\frac{1-\nu}{2} ; \frac{3}{2} ; \frac{z^{2}}{2}\right)\right],
\end{aligned}
$$

where ${ }_{1} F_{1}(a ; b ; x)$ is the confluent hypergeometric function of the first kind as defined in [24, eq. (13.1.2)]. Then, we can write

$$
\begin{aligned}
F(z) & =(2 z)^{-m-1 / 2} \Gamma(2 m+1) \exp \left[\frac{a^{2}}{8 z}\right] \\
& \times\left[D_{-2 m-1}\left(\frac{-a}{\sqrt{2 z}}\right)+D_{-2 m-1}\left(\frac{a}{\sqrt{2 z}}\right)\right] ; \\
& \Re(m)>-1 / 2 \wedge \Re(z)>0 .
\end{aligned}
$$

Now, introducing (10) into (11) we obtain

$$
\begin{gathered}
F(z)=2^{-2 m} \sqrt{\pi} \frac{(2 m) !}{m !} z^{-m-1 / 2}{ }_{1} F_{1}\left(m+\frac{1}{2} ; \frac{1}{2} ; \frac{a^{2}}{4 z}\right), \\
\Re(m)>-1 / 2 \wedge \Re(z)>0 .
\end{gathered}
$$

Therefore, (7) becomes

$$
\begin{aligned}
E_{R^{2}} & \left\{R^{2 n} e^{s R^{2}}\right\}=\frac{1}{2 \pi \sigma_{x} \sigma_{y}} \exp \left[-\frac{\mu_{x}^{2}}{2 \sigma_{x}^{2}}-\frac{\mu_{y}^{2}}{2 \sigma_{y}^{2}}\right] \sum_{k=0}^{n}\left(\begin{array}{l}
n \\
k
\end{array}\right) \\
\times & \mathfrak{L}\left\{x^{k-1 / 2} \cosh \left(\frac{\mu_{x}}{\sigma_{x}^{2}} \sqrt{x}\right) ; \frac{1}{2 \sigma_{x}^{2}}-s\right\} \\
\times & \mathfrak{L}\left\{x^{n-k-1 / 2} \cosh \left(\frac{\mu_{y}}{\sigma_{y}^{2}} \sqrt{x}\right) ; \frac{1}{2 \sigma_{y}^{2}}-s\right\} .
\end{aligned}
$$

Therefore, considering (8) and (12), after some manipulations we obtain

$$
\begin{aligned}
& \phi_{R^{2}}^{(n)}(s)=\frac{1}{\sigma_{y} \sigma_{x} 2^{2 n+1}} \exp \left[-\frac{\mu_{x}^{2}}{2 \sigma_{x}^{2}}-\frac{\mu_{y}^{2}}{2 \sigma_{y}^{2}}\right] \sum_{k=0}^{n}\left(\begin{array}{l}
n \\
k
\end{array}\right) \\
& \quad \times \frac{(2 k) !(2(n-k)) !}{k !(n-k) !}\left(\frac{2 \sigma_{x}^{2}}{1-2 \sigma_{x}^{2} s}\right)^{k+\frac{1}{2}}\left(\frac{2 \sigma_{y}^{2}}{1-2 \sigma_{y}^{2} s}\right)^{n-k+\frac{1}{2}} \\
& \quad \times{ }_{1} F_{1}\left(k+\frac{1}{2} ; \frac{1}{2} ; \frac{\mu_{x}^{2}}{2 \sigma_{x}^{2}\left(1-2 \sigma_{x}^{2} s\right)}\right) \\
& \quad \times{ }_{1} F_{1}\left(n-k+\frac{1}{2} ; \frac{1}{2} ; \frac{\mu_{y}^{2}}{2 \sigma_{y}^{2}\left(1-2 \sigma_{y}^{2} s\right)}\right)
\end{aligned}
$$

Taking into account that, for the parameter values in (14), the confluent hypergeometric function can be expressed, using [25, eq. (07.20.03.0007.01) and (05.08.06.0006.01)], in terms of a finite combination of elementary functions as

$$
\begin{aligned}
& { }_{1} F_{1}(a ; a-n ; z)=\frac{(-1)^{n} e^{z}}{(1-a)_{n}} \\
& \quad \times \sum_{m=0}^{n} \frac{(-n)_{m}(a-n+m)_{n-m}}{m !}(-z)^{m},
\end{aligned}
$$


where $(a)_{n}$ denotes the Pochhammer symbol, and with the help of equalities

$$
\begin{gathered}
\left(\frac{1}{2}+m\right)_{k-m}=\frac{1}{2^{2(k-m)}} \frac{(2 k) !}{k !} \frac{m !}{(2 m) !}, \text { for } k \geqslant m, \\
(-k)_{m}=(-1)^{m} \frac{k !}{(k-m) !}, \text { for } k \geqslant m, \\
\left(\frac{1}{2}-k\right)_{k}=(-1)^{k} \frac{(2 k) !}{k ! 2^{2 k}},
\end{gathered}
$$

which can be easily demonstrated by mathematical induction, after some manipulation we can write

$$
\begin{aligned}
& \phi_{R^{2}}^{(n)}(s)=\frac{1}{\sqrt{\left(1-2 \sigma_{x}^{2} s\right)\left(1-2 \sigma_{y}^{2} s\right)}} \\
& \quad \times \exp \left(\frac{\mu_{x}^{2} s}{1-2 \sigma_{x}^{2} s}+\frac{\mu_{y}^{2} s}{1-2 \sigma_{x}^{2} s}\right) \\
& \quad \times \frac{n !}{2^{2 n}} \sum_{k=0}^{n} \frac{(2 k) !(2(n-k)) !}{k !(n-k) !} \\
& \times\left(\frac{2 \sigma_{x}^{2}}{1-2 \sigma_{x}^{2} s}\right)^{k}\left(\frac{2 \sigma_{y}^{2}}{1-2 \sigma_{y}^{2} s}\right)^{n-k} \\
& \times\left(\sum_{m=0}^{k} \frac{\left(4 z_{x}\right)^{m}}{(k-m) !(2 m) !}\right)\left(\sum_{m=0}^{n-k} \frac{\left(4 z_{y}\right)^{m}}{(n-k-m) !(2 m) !}\right)
\end{aligned}
$$

where $z_{x}=\frac{\mu_{x}^{2}}{2 \sigma_{x}^{2}\left(1-2 \sigma_{x}^{2} s\right)}, z_{y}=\frac{\mu_{y}^{2}}{2 \sigma_{y}^{2}\left(1-2 \sigma_{y}^{2} s\right)}$.

Considering now that $\gamma=R^{2} E_{s} / N_{0}=\left(X^{2}+Y^{2}\right) E_{s} / N_{0}$, the statistics of $\gamma$ will be the same as the ones for $R^{2}$ by just scaling parameters $\mu_{x}, \mu_{y}, \sigma_{x}$ and $\sigma_{y}$ by $\sqrt{E_{s} / N_{0}}$. Therefore, from (19) and using the parameters defined in Section II, after some algebraic manipulation, (5) is obtained.

\section{AVERAGE DETECTION PROBABILITY}

The average probability of detection $\overline{P_{d}}$ of an unknown deterministic signal in the presence of noise in a wireless fading channel can be calculated, assuming that the PDF of the received SNR is denoted by $f_{\gamma}(\gamma)$, as [26]

$$
\overline{P_{d}}=\int_{0}^{\infty} Q_{u}(\sqrt{2 \gamma}, \sqrt{\lambda}) f_{\gamma}(\gamma) d \gamma
$$

where $u=T W$ is the product of the one-side bandwidth $W$ and the observation time interval $T$, which can be easily adjusted to get $u \in \mathbb{N}, \lambda$ is the energy detection threshold and $Q_{u}(.,$.$) is the u^{t h}$ order generalized Marcum- $Q$ function [19, eq. (4.60)],

In the following derivations, we leverage the approach in [5] to obtain an analytical expression for (20), on which using the series expansion of the generalized Marcum- $Q$ function given by

$$
Q_{u}(\sqrt{2 \gamma}, \sqrt{\lambda})=\sum_{n=0}^{\infty} \frac{\gamma^{n} e^{-\gamma}}{n !} \frac{\Gamma(u+n, \lambda / 2)}{\Gamma(u+n)},
$$

the average detection probability can be obtained by combining (21) and (20), yielding

$$
\begin{aligned}
\overline{P_{d}} & =\left.\sum_{n=0}^{\infty} \frac{\Gamma(u+n, \lambda / 2)}{n ! \Gamma(u+n)} \phi_{\gamma}^{(n)}(s)\right|_{s=-1} \\
& =\left.\sum_{n=0}^{\infty} \sum_{q=0}^{u+n-1}\left(\frac{\lambda}{2}\right)^{q} \frac{e^{-\lambda / 2}}{n ! q !} \phi_{\gamma}^{(n)}(s)\right|_{s=-1},
\end{aligned}
$$

where $\phi_{\gamma}^{(n)}(s)$ is the generalized MGF of $\gamma$. Then, the average detection probability of an energy detector in Beckmann fading can be obtained by plugging (5) into (22). The so-called receiver operating characteristic (ROC) curve is obtained by representing $\overline{P_{d}}$ vs. $P_{f}$, for different values of $u$ and $\lambda$, where $P_{f}$ is the false alarm probability (detection, in the absence of signal, of noise which is erroneously considered to be signal), and which is given by [26]

$$
P_{f}=\frac{\Gamma(u, \lambda / 2)}{\Gamma(u)}=e^{-\lambda / 2} \sum_{k=0}^{u-1} \frac{(\lambda / 2)^{k}}{k !},
$$

where $\Gamma(a, x)=\int_{x}^{\infty} t^{a-1} e^{-t} d t$, is the upper incomplete Gamma function and $\Gamma(a)=\Gamma(a, 0)$ is the Gamma function. The last equality in (23) is obtained when $u$ is a positive integer, considering [23, eq. (8.352.2)].

Alternatively, a complementary ROC curve is obtained by representing $\overline{P_{m}}=1-\overline{P_{d}}$, defined as the probability of missed detection (i.e., failing to detect a signal which is present in the channel) vs. $P_{f}$, which can be interpreted in a simple way: the lower the complementary ROC curve, the better system performance for energy detection.

\section{A. Average detection probability with diversity combining}

In the previous analysis, we assumed a single-antenna configuration for the energy detection receiver. We now study the effect of using maximal ratio combining (MRC) and squarelaw combining (SLC) on the energy detection performance, considering receivers equipped with $L$ antennas.

1) Maximal Ratio Combining (MRC): In MRC, the instantaneous combined SNR is given by $\gamma_{M R C}=\sum_{k=1}^{L} \gamma_{k}$, where $\gamma_{k}$ is the instantaneous SNR at the $k$ branch. Assuming that the receive signals at every branch are independent, with the help of the multinomial theorem we can write

$$
\phi_{M R C}^{(n)}(s)=\sum_{\tau(n, L)} \frac{n !}{q_{1} ! q_{2} ! \cdots q_{L} !} \phi_{\gamma_{1}}^{\left(q_{1}\right)}(s) \phi_{\gamma_{2}}^{\left(q_{2}\right)}(s) \cdots \phi_{\gamma_{L}}^{\left(q_{L}\right)}(s) \text {, }
$$

where $\tau(n, L)$ is defined as the set of $L$-tuples such that $\tau(k, L)=\left\{\left(q_{1}, q_{2}, \cdots, q_{L}\right): q_{m} \in \mathbb{N}, \sum_{m=1}^{L} q_{m}=k\right\}$. Combining now (22) and (24), $\overline{P_{d}}$ becomes

$$
\begin{gathered}
\overline{P_{d, M R C}}=\left.\sum_{n=0}^{\infty} \frac{\Gamma(u+n, \lambda / 2)}{n ! \Gamma(u+n)} \phi_{M R C}^{(n)}(s)\right|_{s=-1}= \\
\left.\sum_{n=0}^{\infty} \sum_{\tau(n, L)} \sum_{m=0}^{u+n-1} \frac{\left(\frac{\lambda}{2}\right)^{m} e^{-\lambda / 2}}{m !} \frac{\phi_{\gamma_{1}}^{\left(q_{1}\right)}(s)}{q_{1} !} \cdots \frac{\phi_{\gamma_{L}}^{\left(q_{L}\right)}(s)}{q_{L} !}\right|_{s=-1},
\end{gathered}
$$




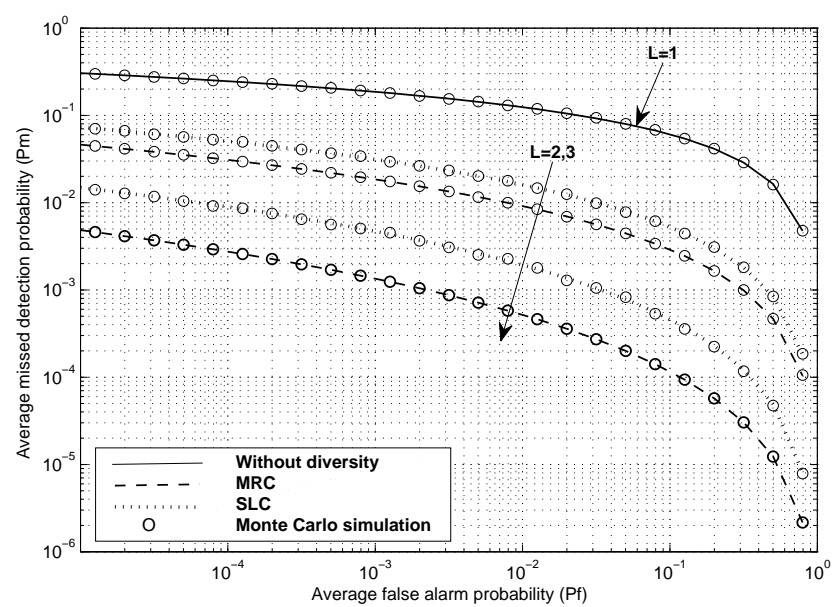

Figure 1. Complementary ROC curve under Beckmann fading for different numbers of receive diversity branches $L$ and sampling policies. Parameters values: average SNR per branch $\bar{\gamma}_{k}=15 \mathrm{~dB} ; q=0.5 ; K=2 ; r=1$; $u=5$.

where in the last equality we assumed that $u \in \mathbb{N}$. Under this scheme, $P_{f}$ is also given by (23).

2) Squared Law Combining (SLC): This is a post-detection combining method, that is, the decision variable is combined after sampling [22, sec. 5.4]. Again, $\gamma_{S L C}=\sum_{k=1}^{L} \gamma_{k}$ as in the MRC case, therefore $\phi_{S L C}^{(n)}=\phi_{M R C}^{(n)}$ as given in (24). However, the number of samples to be considered is not $u$ but $L u$. Hence, $\overline{P_{d}}$ and $P_{f}$ are obtained, respectively as in (25). and (23). by just substituting $L$ by $L u$.

\section{NUMERICAL RESULTS}

Complementary ROC curves are represented in this section for different fading parameters and diversity schemes. For the sake of simplicity in the discussion, we assume (independent identically distributed (i.i.d.) signals, although this restriction is not necessary in our framework.

In Fig. 1 we can see the influence of the number of diversity branches in the energy detection performance, as well as the influence of the sampling policy. The ROC curves decrease in a very appreciable manner when the number of branches increases. For a given receive array size, the MRC strategy shows a better behavior than SLC, at the expense of requiring a precise channel knowledge. Also, as the number of diversity branches increases, the more significant is the improvement provided by MRC. We have performed Monte Carlo simulations in order to validate the theoretical results, which are included in the figures with marker dots, and show a perfect agreement.

In Fig. 2 the influence of fading severity is evaluated. As expected, the probability of a wrong detection is much lower in the high-SNR regime $\left(\bar{\gamma}_{k}=25 \mathrm{~dB}\right)$, but it is relevant how the fading severity and the sampling policy increment their influence at this regime. Conversely, in the low SNR-regime $\left(\bar{\gamma}_{k}=5 \mathrm{~dB}\right)$, the differences are smaller. Indeed, MRC shows again a better performance than the SLC policy.

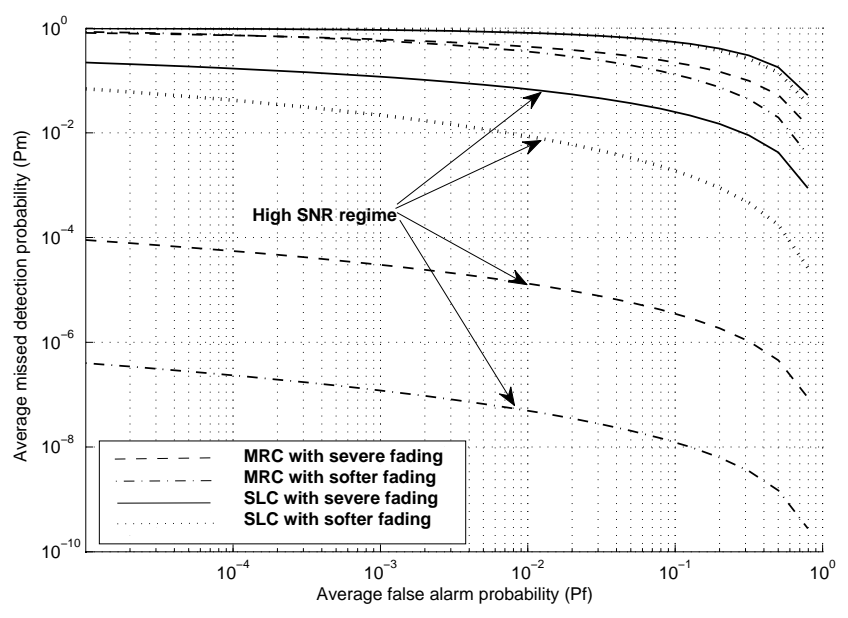

Figure 2. Complementary ROC curve under Beckmann fading for different values of fading severity parameters and average SNRs. 'Severe fading': $\{q=$ $0.1 ; K=0.1\}$; 'Softer fading': $\{q=0.9 ; K=3\}$. Analogously, 'High SNR': $\bar{\gamma}_{k}=25 \mathrm{~dB}$; 'Low SNR'. $\bar{\gamma}_{k}=5 \mathrm{~dB}$. Parameters values: $L=3$; $r=1 ; u=5$.

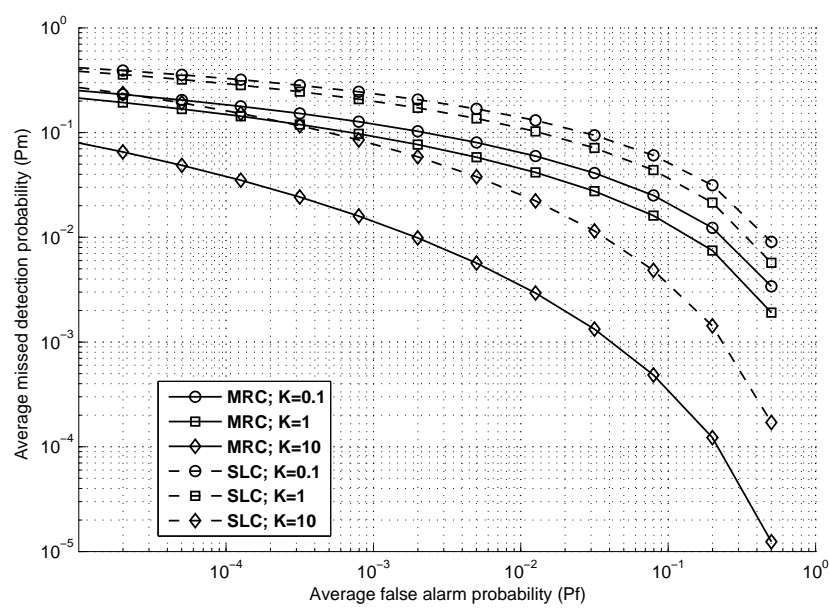

Figure 3. Complementary ROC curve under Beckmann fading for different values of $K$. Parameters values: average SNR per branch $\bar{\gamma}_{k}=10 \mathrm{~dB} ; L=3$; $r=0.7 ; q=0.5$, and $u=5$.

Fig. 3 and Fig. 4 explore the influence of parameters $K$ and $r$, respectively. Fig. 3 shows how the presence of a strong Line-of-Sight (LOS), represented by $K=10$, substantially improves the performance. Fig. 4 shows that the best detection performance is attained when the average in-phase and quadrature LOS powers are equal, $r=1$, whereas the performance is degraded as $r$ is decreased. This is coherent with the fact that the parameter $r$ affects fading severity in a similar way as the Hoyt parameter $q$.

\section{Conclusions}

In this work, we analyzed the performance of different multi-branch energy detectors in the presence of Beckmann fading. Thanks to the novel closed-form expression for the 


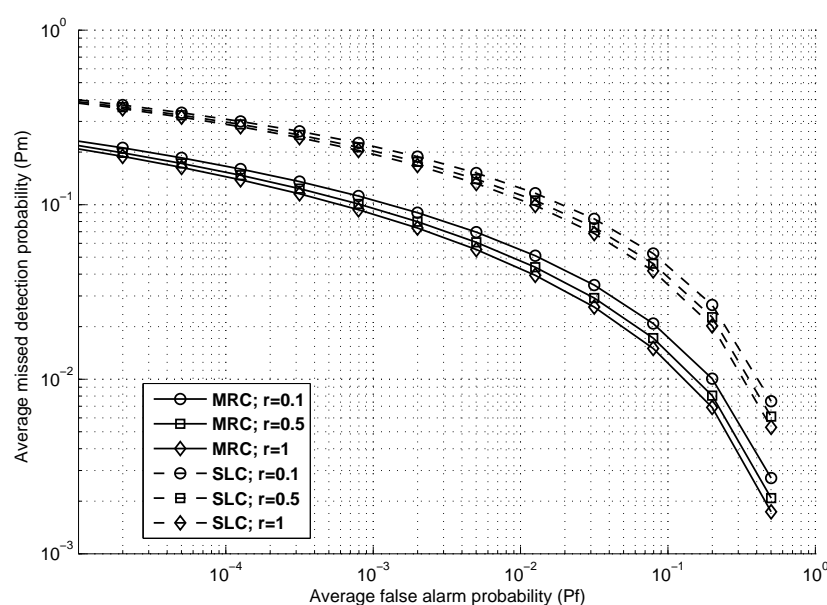

Figure 4. Complementary ROC curve under Beckmann fading for different values of $r$. Parameters values: average SNR per branch $\bar{\gamma}_{k}=10 \mathrm{~dB} ; L=3$; $K=1 ; q=0.5$, and $u=5$.

generalized MGF of the received SNR under Beckmann fading here derived, analytical expressions for the probability of detection were obtained with similar complexity to those available for Rician or Hoyt fading. The ROC curves corresponding to pre-detection (MRC) and post-detection (SLC) combining strategies have been obtained as an infinite sum of elementary functions with a reasonably fast convergence. As a general conclusion, any unbalance between the underlying Gaussian RVs inherent to Beckmann fading, either in the LOS (parameter $r$ ) or diffuse (parameter $q$ ) parts, has a negative impact on the detection probability compared to the balanced cases $(r=1$ or $q=1)$.

\section{ACKNOWLEDGMENT}

The work of Juan P. Peña-Martín and Juan M. Romero-Jerez was supported by the Spanish Government-FEDER public Project No. TEC2013-42711-R and Universidad de Malaga - Campus de Excelencia Andalucia-Tech. The work of F.J. Lopez-Martinez was funded by Junta de Andalucia (P11-TIC7109), Spanish Government-FEDER (TEC2013-44442-P) and Universidad de Malaga - Campus de Excelencia AndaluciaTech.

\section{REFERENCES}

[1] J. Mitola and G. Q. Maguire, "Cognitive radio: making software radios more personal," IEEE Personal Communications, vol. 6, no. 4, pp. 1318, Aug 1999.

[2] A. Goldsmith, S. A. Jafar, I. Maric, and S. Srinivasa, "Breaking spectrum gridlock with cognitive radios: An information theoretic perspective," Proc. IEEE, vol. 97, no. 5, pp. 894-914, May 2009.

[3] H. Urkowitz, "Energy detection of unknown deterministic signals," Proceedings of the IEEE, vol. 55, no. 4, pp. 523-531, April 1967.

[4] F. Digham, M.-S. Alouini, and M. K. Simon, "On the energy detection of unknown signals over fading channels," IEEE Trans. Commun., vol. 55, no. 1, pp. 21-24, Jan. 2007.

[5] A. Annamalai, O. Olabiyi, S. Alam, O. Odejide, and D. Vaman, "Unified analysis of energy detection of unknown signals over generalized fading channels," in Wireless Communications and Mobile Computing Conference (IWCMC), 2011 7th International, July 2011, pp. 636-641.

[6] P. C. Sofotasios, E. Rebeiz, L. Zhang, T. A. Tsiftsis, D. Cabric, and S. Freear, "Energy Detection Based Spectrum Sensing Over $\kappa-\mu$ and $\kappa$ $\mu$ Extreme Fading Channels," IEEE Trans. Veh. Technol., vol. 62, no. 3 , pp. 1031-1040, March 2013.

[7] A. Annamalai and A. Olaluwe, "Energy detection of unknown deterministic signals in $\kappa-\mu$ and $\eta-\mu$ generalized fading channels with diversity receivers," in Computing, Networking and Communications (ICNC), 2014 International Conference on, Feb 2014, pp. 761-765.

[8] E. Chatziantoniou, B. Allen, and V. Velisavljevic, "Threshold optimization for energy detection-based spectrum sensing over hyper-Rayleigh fading channels," IEEE Commun. Lett., vol. 19, no. 6, pp. 1077-1080, Jun. 2015.

[9] Y. Alghorani, G. Kaddoum, S. Muhaidat, and S. Pierre, "On the Approximate Analysis of Energy Detection Over $n *$ Rayleigh Fading Channels Through Cooperative Spectrum Sensing," vol. 4, no. 4, pp. 413-416, Aug. 2015.

[10] P. C. Sofotasios, L. Mohjazi, S. Muhaidat, M. Al-Qutayri, and G. K. Karagiannidis, "Energy Detection of Unknown Signals Over Cascaded Fading Channels," IEEE Antennas Wireless Propag. Lett., vol. 15, pp. 135-138, 2016.

[11] P. Beckmann and A. Spizzichino, The Scattering of Electromagnetic Waves from Rough Surfaces, 2nd ed. Boston, MA: Artech House, 1987.

[12] H. T. Dougherty, A survey of microwave fading mechanisms remedies and applications. Wave Propagation Laboratories, 1968, vol. 52.

[13] Y. Xie and Y. Fang, "A general statistical channel model for mobile satellite systems," IEEE Trans. Veh. Technol., vol. 49, no. 3, pp. 744752, May 2000.

[14] N. Youssef, C.-X. Wang, M. Patzold, I. Jaafar, and S. Tabbane, "On the statistical properties of generalized Rice multipath fading channels," in Vehicular Technology Conference, VTC 2004-Spring. 2004 IEEE 59th, vol. 1, May 2004, pp. 162-165.

[15] G. A. Ropokis, A. A. Rontogiannis, P. T. Mathiopoulos, and K. Berberidis, "An Exact Performance Analysis of MRC-OSTBC over Generalized Fading Channels," IEEE Trans. Commun., vol. 58, no. 9, pp. 2486-2492, Sep. 2010.

[16] W. Dahech, N. Hajri, N. Youssef, T. Kawabata, and M. Pätzold, "Outage statistics for Beckmann fading channels in non-isotropic scattering environments," in 21st Asia-Pacific Conference on Communications (APCC) 2015, Oct. 2015, pp. 164-168.

[17] B. Zhu, J. Cheng, N. Al-Dhahir, and L. Wu, "Asymptotic Analysis and Tight Performance Bounds of Diversity Receptions Over Beckmann Fading Channels With Arbitrary Correlation," IEEE Trans. Commun., vol. PP, no. 99, pp. 1-1, 2016.

[18] P. Beckmann, "Statistical distribution of the amplitude and phase of a multiply scattered field," JOURNAL OF RESEARCH of the National Bureau of Standards-D. Radio Propagation, vol. 66D, no. 3, pp. 231240, 1962.

[19] M. K. Simon and M.-S. Alouini, Digital Communications over Fading Channels, 2nd ed. John Wiley \& Sons, Inc., 2005.

[20] M. D. Yacoub, G. Fraidenraich, H. B. Tercius, and F. C. Martins, "The symmetrical $\eta-\kappa$ distribution: a general fading distribution," IEEE Trans. Broadcast., vol. 51, no. 4, pp. 504-511, Dec. 2005.

[21] _ , "The asymmetrical $\eta-\kappa$ distribution," Journal of Communication and Information Systems, vol. 20, no. 3, pp. 182-187, 2005.

[22] J. G. Proakis, Digital Communications, 4th ed. McGraw Hill, 2001

[23] I. S. Gradshteyn and I. M. Ryzhik, Table of Integrals, Series and Products, 6th ed. Academic Press, 2000.

[24] M. Abramowitz and I. A. Stegun, Handbook of Mathematical Functions with Formulas, Graphs, and Mathematical Tables, 10th ed. U.S. Department of Commerce - N.B.S., Dec. 1972.

[25] "The wolfram function site," Wolfram Research, Inc., 2016. [Online]. Available: http://functions.wolfram.com/

[26] F. Digham, M.-S. Alouini, and M. K. Simon, "On the energy detection of unknown signals over fading channels," in Proc. IEEE International Conference on Communication. ICC '03., vol. 5, May 2003, pp. 35753579 . 